\title{
Analysis of Semantic Information via Information Reports
}

\author{
Julio Ostalé
}

Universidad de Salamanca (PhD Programme), Spain. E-mail: ostale@yahoo.es

\begin{abstract}
Semantic information is analysed by means of two consecutive approaches. Firstly, we consider semantic information via ordinary-language reports of the form " $X$ carries the information that $Y$ ". Secondly, and partially based on the previous analysis, we obtain a number of a priori conditions a physical system has to fulfill for semantic information to take place in such a system.
\end{abstract}

Keywords: semantic information, information reports, a priori conditions, situation theory, channel theory, semantics, objectivity problem

Acknowledgement: The author carries out his research within the project "Las lógicas de la red" (ref. HUM2006-12848C02-01), supported by the Spanish "Ministerio de Educación y Ciencia". He is also indebted to Francisco Salto Alemany and José María Díaz Nafría for encouraging him to take part into the "First International Meeting of Experts in Information Thepries" held in León (Spain), during November $6^{\text {th }}-7^{\text {th }} 2008$.

W hat is semantic information? This is after all the topic we are going to deal with. Notice that, even before we propose a definition of semantic information, our choice of the very term "semantic information" apparently involves two things: (i) because of the English grammar, our choice should imply that there is something we recognize as information, of which semantic information is but a variety; (ii) because of the well established semiotic distinction syntaxsemantics-pragmatics, it should also imply that there are two further varieties of information, namely syntactic information and pragmatic information. Now, I will neither accept nor deny theses (i) and (ii). The reason I have put the expression "semantic information" in the title of this paper is that I wish to focus on certain topic, indeed a very old one, that in the last sixty years has been discussed under the umbrella of that expression. "Semantic information" is used as a label for those inquiries that try to merge ideas from Shannon's Statistical Theory of Signal Transmission with some theory of meaning.

\section{Information Reports}

A neutral formulation of our topic is given in the major work of Channel Theory: "How is it possible for one thing to carry information about another?" (Barwise and Seligman, 1997: xi). Channel Theory, whose philosophical background is consistent with the ideas of this paper, is one of the most recent descendants of Situation Theory, a mathematical logic-oriented theory of information first developed in Barwise and Perry (1983). The general framework of Situation Theory, including terminology and basic hypotheses, was fixed by Devlin (1991). Actually, the picture is much more complicated once we observe some facts: there are different versions of Situation Theory in the literature; the standard Channel Theory as developed in Barwise and Seligman (1997) stems from an earlier version that was also called Channel Theory, see Moss and Seligman (1994); finally, relationships between different versions of Situation Theory and different versions of Channel Theory are not completely clear. Our considerations in this paper will run in parallel 
with those ideas that are commonplaces to every version of Situation Theory and every version of Channel Theory.

What does it mean that some things carry information about each another? We know that fire causes smoke; this (causal) regularity or constraint supports in some way the fact that every piece of smoke carries the information that there is a fire; however, and this is the controversial point, the semantic relation denoted by "carries the information that" is usually thought of as different from the nomic relation denoted by "causes". In other words, we figure out and speak of the world as if there were information flow out there; furthermore, this information flow is taken as something that rests on regularities, although it is not a regularity by itself. The main problem is how to achieve a good explanation for information on the basis of regularities.

We are going to work out an indirect notion of information guided by the use of language. In which sense? The nature of information is not given by English language, of course, but we can substitute the abstract notion of information by a more concrete notion with the help this language. The key is to identify typical reports describing what we are interested in, and then to define our topic as the reference of those reports. Let us start. By an "information transfer" I suggest to understand hereafer anything whose typical linguistic expression is

\section{$X$ carries the information that $Y$}

or some other variant close enough to this one. We will say in the sequel that $X$ is the "source" and $Y$ is the "target" of the transfer. At the linguistic level, statements like (1a) are called "information reports" after Israel and Perry (1990). Typical examples of information transfers are: dark clouds in the sky bear the information that it is going to rain nearby, the open door of my house carries the information that someone is in there, your smiling brings the information that you are happy, a jacket in my brother's wardrove means that my brother owns the jacket, and so on. Of course, there are also more complex information phenomena; to put an example, the word "open" on the door of a shop indicates that we can enter the shop, though this permission is not something we can easily recognize as a state of affairs. For convenience, we consider in this paper only those information transfers in which both $X$ and $Y$ are state of affairs we can easily describe in terms of "item $s$ in of the type T". In the previous example, it is far from clear whether the item is a concrete person, any person, all the persons, the situation itself, the relation of a person with respect to the shop, etc.

In ordinary language, while keeping the term "information" playing the role of a direct object, we can distinguish two groups of expressions reductible to (1a). Firstly, there are those expressions whose main verb is static, in the sense that doesn't refer to change, and indicates possession: $X$ conveys the information that $Y, X$ supports the information that $Y, X$ has the information that $Y, X$ gives you the information that $Y$, etc. Secondly, there are those expressions whose main verb is dynamic and indicates transportation: $X$ bears the information that $Y$, $X$ brings the information that $Y, X$ transports the information that $Y$, etc. This distinction is admittedly not very sharp in everyday conversations; nevertheless, it will become quite useful for philosophical analysis. Further, there are expressions akin to (1a) without the term "information": $X$ informs that $Y, X$ reports that $Y, X$ means that $Y, X$ involves $\mathrm{Y}$, or $\mathrm{X}$ implies $\mathrm{Y}$.

There is the habit of assuming $X$ and $Y$ to be of different nature. It is frequently accepted that $X$ stands for something physical, the socalled "signal", whereas $Y$ stands for something either mental or abstract, the socalled "information content of the signal". For some reason, schema (1a) is assimilated to the schema of propositional attitudes: A believes that $p, A$ sees (that) $p, A$ truthfully asserts that $p, A$ remembers that $p, A$ wonder wether $p$, and so on. This is the case for instance in Israel and Perry (1990), a brief article whose influence can be traced until important monographs like Pérez (2000) or Floridi (2004). But is this the only way for an understanding of the above schema? We would like to show that the similarity between (1a) and propositional attitudes is misleading, hence a potential source of pseudo-problems.

By saying or writing first $X$, then a verb, and then $Y$ preceded by the word "information", it 
seems to many authors that $Y$ is qualified in a deep sense that $X$ is not. To complicate matters, this linguistic distinction between a subject whose form is " $X$ " and a direct object whose form is "the information that $Y$ " meets to quickly a far from clear intuition involving the double nature of information, namely the intuition by which information involves physical ingredients (signals, documents, TV screens, pictures...) as well as ingredients that are not physical (meanings, contents, propositions...). In abstract thinking, the encounter of two dichotomies (A versus B) and ( $M$ versus $N$ ) too frequently yields to a new dichotomy ( $A$ or $M$ ) versus ( $B$ or $N$ ). In our case, the above linguistic distinction together with the above intuition behave like that. By means of a one-to-one implicit attribution, many authors come to think that $X$ stands for something physical whereas $Y$ stands for something non-physical. They interpret (1a) by saying that the signal $X$ conveys the informational content $Y$. Yet this assimilation is too rude, even if we accept the token-type dimension of every information transfer. If you rephrase (1a) by the synonymous

\section{$X$ informs that $Y$}

then you begin to have reasons for being suspicious about the analogy between (1a) and propositional attitudes. With (1b) it is not so clear that $Y$ has to be a proposition. Actually, the original meaning of the verb, by which informing boils down to imposing a form over an object (see Capurro, 1978), suggests a relation between two entities of the same sort. And more importantly, (1a) and (1b) can sometimes be reversed into.

\section{$Y$ carries the information that $X$}

This transformation does not always make sense. An example for the possibility of such a transformation: snow on the roof of your house bears the information that it is snowing, and the fact that it is snowing informs that there must be snow on the roof of your house. A counterexample: the door of my house being open informs that someone is in there, but someone being at home does not usually carry the information that the door is open. Now, the important thing is not under which conditions (1b) can be transformed into (1c).
The point here is that the mere possibility of such a transformation falsifies the analogy between (1a) and propositional attitudes, since there is no way of reversing for instance $A$ believes that $p$ into $p$ believes that $A$.

This is plenty of consequences. Consider e.g. that an important discussion when interpreting $Y$ in (1b) as a proposition concerns veridicality. Some authors defend that the information content of a signal is a true proposition, as in the case of $p$ in $A$ knows that $p$. Others maintain that the information content of a signal is a proposition that can be either true or false, as in the case of $p$ in $A$ believes that $p$. However, both thesis share that the information content of a signal is a proposition. If we interpret $Y$ as something distinct of a proposition, the debate on veridicality calls for a new perspective. Let us consider an argument by analogy. I consider that (1b) is closer to (2b) than to (2a) in these examples:

$$
\text { John is } 90 \mathrm{~kg} \text { weight. }
$$

Body $a$ is attracted by the gravitational field of body $b$.

In (2a) we have a sentence whose subject refers to a concrete entity, John, whereas the object refers to an abstract entity, $90 \mathrm{~kg}$ weight. In (2b) the difference between how the subject and the object are expressed, putting "gravitational field" only before the object, does not constitute a big obstacle to our understanding of gravitation as a kind of relation between bodies. It is obvious that we interpret (2b) as reporting a relation between two bodies $a$ and $b$, not as reporting a relation between the body $a$ and the gravitational field of $b$. Likewise, I propose to understand the subject and object of (1a) as entities of a same species. Not necessarily spatiotemporal entities, though.

To refuse that $Y$ is a proposition, the socalled "information content", encoded by some entity or event $X$, the so-called "signal", is just an argument for the plausibility of this claim: $X$ and $Y$ are of the same nature. Well, but what exactly are $X$ and $Y$ ? 


\section{A Priori Conditions}

It is time to ask for the internal structure of both the source and the target of an information transfer. And the first thing worth saying is that, following Grice (1957) and Situation Theory, entities carry information about each other as long as they are in an abstract state. It is not the bare cloud that carries information whatsoever, but rather the cloud-being-dark (as opposed to the cloudbeing-white) that carries the information of rain. Just think of it by reductio ad absurdum: something that cannot be but in one abstract state remains indifferent to environmental changes, hence its being in that state can never be a source of information. This interplay between concrete entities and abstract states or types is of the biggest importance in understanding the structure of information transfers.

The distinction of tokens (concrete bearers of information) and types (abstract states in which tokens can be) is dependent of some classification schema. In Channel Theory we write $\left\{s_{1}, s_{2} \ldots\right\}$ and $\left\{T_{1}, T_{2} \ldots\right\}$, respectively, for tokens and types of certain classification schema. Then we can write $s: T$ to denote that token $s$ is of type $T$. Accordingly, two classifications are two structures of the form

$$
\begin{aligned}
& \mathbf{A}=\left(\left\{a_{1}, a_{2} \ldots\right\},\left\{A_{1}, A_{2} \ldots\right\},:^{\mathrm{A}}\right) \\
& \mathbf{B}=\left(\left\{b_{1}, b_{2} \ldots\right\},\left\{B_{1}, B_{2} \ldots\right\},:^{\mathrm{B}}\right)
\end{aligned}
$$

We omit superscripts in $:^{A}$ and $:^{B}$, and we take arbitrary $a$ from $\left\{a_{1}, a_{2} \ldots\right\}, A$ from $\left\{A_{1}\right.$, $\left.A_{2} \ldots\right\}, b$ from $\left\{b_{1}, b_{2} \ldots\right\}$, and $B$ from $\left\{B_{1}, B_{2} \ldots\right\}$. It makes now sense to say that it is a specific token $a$ as being of type $A$ that can carry information about another token $b$ as being of type $B$. In symbols, $a$ : $A$ carries information about $b: B$. Actually, we should better say that it is a specific token $a$ as being of type $A$ that can carry the information that another token $b$ is of type $B$. Again in symbols, $a: A$ carries the information that $b: B$. The second manner is better in that it avoids the notion of information as something different from the ordered pair $b: B$. Hence, schema (1b) amounts to:

$$
a: A \text { informs that } b: B
$$

Ordered pairs like $a: B$ can be called "information units". From a different perspective, Devlin (1991) obtains a schema almost identical to (1d) for which he applies a terminology that has become standard in Situation Theory: a supports $A, b$ supports $B$, and $a$ carries the information that $B$. (We can accept to some extent this terminology, although we also have to be conscious about the fact that Situation Theory, as opposed to Channel Theory, does not consider relations between tokens; that's why the relation of carrying information do not take into account token b.) By the way, notice that Devlin's distinction between two senses in which a token relates to a type matches pretty well our previous distinction between static and dynamic verbs for information reports; to support and to carry relate side by side to static and dynamic verbs in information reports.

By paying attention to the previous analysis, we obtain two conditions a complex system has to fulfill for information transfer to take place between two of is parts (modelled by $\mathbf{A}$ and $\mathbf{B}$ ).

(Condition 1) For one token $a$ to be the source of some information transfer, it is required that $a$ can be classified by more than one type. To be precise, in order to inform about $n$ different things it has to admit at least $n$ different types $A_{1}, \ldots A_{n}$.

(Condition 2) Given that $a$ is the source of an information transfer, for $b$ to be the target of the very same transfer, it is required that $b$ can be classified by more than one type. To be precise, in order $b$ to convey as much information as $a$ it has to admit at least $n$ different types $B_{1}, \ldots B_{\mathrm{n}}$.

Lyre (2002) and Szaniawski (1984) have similar observations on the a priori foundations of information flow. The key idea, up to now, is to settle those conditions a system has to hold for information transfers to occur (or not) within the system. Brackets are important here. What we have distilled are just a priori conditions that parts of a system, modelled by $\mathbf{A}$ and $\mathbf{B}$, candidates for being the source and the target of an information transfer, have to possess. But the potentiallity 
of information will not be actuallized unless something links $\mathbf{A}$ to $\mathbf{B}$.

So we have to put in a broader context the source and the target of any information transfer. It is clear that information is neither the property of a given source nor a relation between an isolated source and an equally isolated target. It is rather something that occurs between two different information units that in turn belong to two different classifications. We get:

(Condition 3) For a token $\boldsymbol{a}$ in $\mathbf{A}$ to be informative about a token $b$ in $\mathbf{B}$ within a system, it is required that at least one type of $a$ is regularly related to at least one type of $b$ by means of some information channel $C$ between $\mathbf{A}$ and $\mathbf{B}$.

This condition is the most difficult one to formalize. Indeed, last versions of Situation Theory and the two existing versions of Channel Theory do their best in order to provide a formal account of regularities between types (Situation Theory) or classifications (Channel Theory). In either case, we have to transform (1d) into

$$
\begin{aligned}
& a: A \text { informs that } b: B \text { with } \\
& \text { respect to some channel } C
\end{aligned}
$$

where the exact nature of channel $C$ is to be determined. It plays for information flow the same role that a reference frame plays for the study of movement. And we expect two things: it should be an objetive subtitute of notions like those of agent and knowledge, and it should illuminate classical problems of information flow.

Which problems are classical in the study of information flow? Do other problems reduce to classical problems? We have in mind two of these classical problems. In the first place, we have the informational phenomenon of fallibility, by which sometimes $X$ does not inform that $Y$ although it uses to. In the second place, there is the problem of relativity, by which $X$ informs that $Y_{1}$ ot that $Y_{2}$ depending of the channel (to use our own terminology) being adopted.

\section{Conclusion}

These pages can be seen as an attempt of giving philosophical foundation to Channel Theory. To be more concrete, we have undertaken the task of analysing "the grammar of information flow" (Barwise and Seligman, 1997: 12-13) in order to provide sound philosophical basis to the main proposals of Channel Theory (Barwise and Seligman, 1997: 35, 183)

By analyzing information reports we concluded the symmetry (at ontological level, though not at linguistic level) between their respective sources and targets. That the target of an information transfer is a proposition has been discussed.

By searching the conditions of possibility of information transfers, which are the intended interpretations of information reports, we have found that every source and every target exhibits a token-type duality that rests upon some classificatory schema. Furthermore, semantic linkages between sources and targets seem to depend on some higher-level linkage $C$ between classificatory schemas. As an open problem, the phenomena of fallibility and relativity of information should be faced after defining $C$.

\section{References}

Barwise, J., Perry, J. (1983). Situations and Attitudes. Cambridge: The MIT Press / Bradford.

Barwise, J., Seligman, J. (1997). Information flow. Cambridge: Cambridge University Press.

Capurro, R. (1978). Information. Ein Beitrag zur etymologischen und ideengeschichtlichen Begründung des Informationsbegriffs. München: Saur.

Devlin, K. (1991). Logic and Information. Cambridge: Cambridge University Press.

Floridi, L. (2004). "Information". In L. FLORIDI (ed.). The Blackwell Guide to the Philosophy of Computing and Information, pp. 40-61. Oxford: Blackwell. 
Grice, P. (1957). Meaning. Philosophical Review, 66, 377-388.

Israel, D., Perry, J. (1990). What is information?. In P. HANSON (ed.). Information, Language and Cognition, pp. 1-19. Vancouver: University of British Columbia Press.

Lyre, H. (2002). Informationstheorie. Eine philosopisch-naturwissenschaftliche Einführung. München: Wilhelm Fink.

Moss, L., Seligman, J. (1994). Classification domains and information links: A brief survey. In J. van Eijk, A. Visser (eds.). Logic and Information Flow, pp. 112-124. Cambridge (MA): The MIT Press.

Pérez, M. (2000). El fenómeno de la information. Una aproximación conceptual al flujo informativo. Madrid: Trotta. [English version: Pérez-Montoro, M. (2007). The Phenomenon of Information. Maryland: Scarecrow Press.]

Szaniawski, K. (1984). On defining information. Philosophia Naturalis, 3(4), 244-249.

\section{About the Author}

Julio Ostalé

Bachelor and Master in Philosophy ("Licenciado en Filosofía", old-style 300 ECTS curriculum) by the University of Salamanca. Main editor of the on-line journal Factótum (www.revistafactotum.com). In 2009 is about to finish both a MA in Humanities by Universitat Oberta de Catalunya and a MSc in Logic by the ILLC of the Universiteit van Amsterdam. His field of research is philosophical logic and the philosophy of information. 